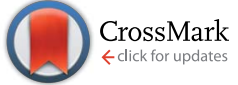

Cite this: RSC Adv., 2016, 6, 32098

\title{
Photoinitiated thiol-epoxy addition for the preparation of photoinduced self-healing fatty coatings
}

\author{
Yu Hui Zhao, Daniela Vuluga, Laurence Lecamp* and Fabrice Burel \\ Herein, we report the use of the photoinitiated thiol-epoxy chemistry for the preparation of a new \\ biosourced self-healing coating. Epoxidized cottonseed oil is used as the main reagent (>80 wt\%) and \\ photocrosslinked in the presence of 0.25 equivalents of 7-mercapto-4-methyl coumarin, used as \\ a photodimerizable and healable reactant. The reaction is initiated with $2 \mathrm{wt} \%$ of a photobase generator \\ (PBG). The stability of the thiol-epoxy mixture under storage and the oxygen-promoted photoinitiating \\ system are some of the advantages illustrated in this study. The physicochemical properties of the \\ resulting coatings as well as the photoreversibility of the coumarin derivative once grafted on the \\ vegetable oil are studied. The photoinitiation step is demonstrated to be efficient for the grafting of the \\ healing reagent as well as for epoxy homopolymerization.
}

Received 9th February 2016

Accepted 22nd March 2016

DOI: $10.1039 / \mathrm{c} 6 \mathrm{ra03693g}$

www.rsc.org/advances

groups such as coumarine, ${ }^{17,18}$ anthracene, ${ }^{19}$ trithiocarbonate

\section{Introduction}

Nowadays, click chemistry involving thiol molecules is widely used for the functionalization of molecules bearing carboncarbon double bonds, the preparation of polymers, or the crosslinking of polymer materials. Although this chemistry can be applied on all carbon-carbon unsaturation types, this particular type usually requires high thiol amounts compared to ene molecules, long reaction times and leads to poor yield when applied to fatty double bonds, due to their low reactivity and numerous side reactions..$^{1-4}$ On the other side, addition of thiol on epoxy groups can be an interesting alternative pathway. Thus, the thiol-epoxy reaction is generally used to prepare adhesives or plastic hardeners at industrial scale by thermal methods. ${ }^{5}$ Recently, this reaction has attracted lots of attention in polymer chemistry as both hydroxy and thioether groups can be formed at once and even be further transformed. ${ }^{6,7}$ This reaction may be initiated by a tertiary amine and has been already used in few works to successfully prepare polymer materials. ${ }^{8-10}$ Surprisingly, the thiol-epoxy reaction under UV was scarcely studied until now. Literature only reports the recent development of convenient photobase generators (PBG) for such a reaction..$^{911-16}$ Hence, to the best of our knowledge, this photoinitiated reaction has never been used until now to prepare polymer materials.

The elaboration of photo-healable materials has already been the subject of several works. The photo-healing property is mostly brought by photosensitive molecules or functional

Normandie Université, INSA de Rouen, CNRS UMR 6270, FR 3038, Avenue de l'Université, F-76801 Saint Etienne Du Rouvray Cedex, France. E-mail: laurence. lecamp@insa-rouen.fr units, .... ${ }^{20}$ These photosensitive moieties are grafted on synthetic or natural macromolecular chains, ${ }^{17,19}$ or introduced in a (co-)monomer before its incorporation in a polymer network. ${ }^{18,20}$ The synthesis process of such materials therefore requires at least two steps.

This paper proposes a one-step process to prepare a photohealable coating from epoxidized vegetable oil by using the photoinitiated thiol-epoxy addition. Epoxidized cottonseed oil (ECO) was chosen as raw material and 7-mercapto-4-methylcoumarin (MMC) was selected as photo-healing reagent. Indeed, the coumarin molecule is well known for its reversible dimerization under UV irradiation. ${ }^{21-24}$ The selected coumarin derivative could be grafted onto epoxidized triglycerides thanks to its $\mathrm{SH}$ group. Hence, the thiol-epoxy addition and the dimerization of coumarin groups are expected concomitant under UV irradiation to produce an original photocrosslinked and photo-healable fatty coating (Scheme 1).

This work is presented in two parts. In the first part, the influence of oxygen, photoinitiating system amount and thiol/ epoxy ratio on the photoinitiated thiol-epoxy reaction kinetics will be studied. The second part will consist in the characterization of the coating prepared according to the determined optimal conditions in terms of physico-chemical properties as well as evaluation of photo-healing ability.

\section{Experimental}

\section{Materials}

Epoxidized cottonseed oil was kindly prepared by the Laboratoire de Sécurité des Risques Chimiques (LSPC, INSA de Rouen, France). ${ }^{25}$ 7-Mercapto-4-methyl-coumarin (MMC, 97\%) 


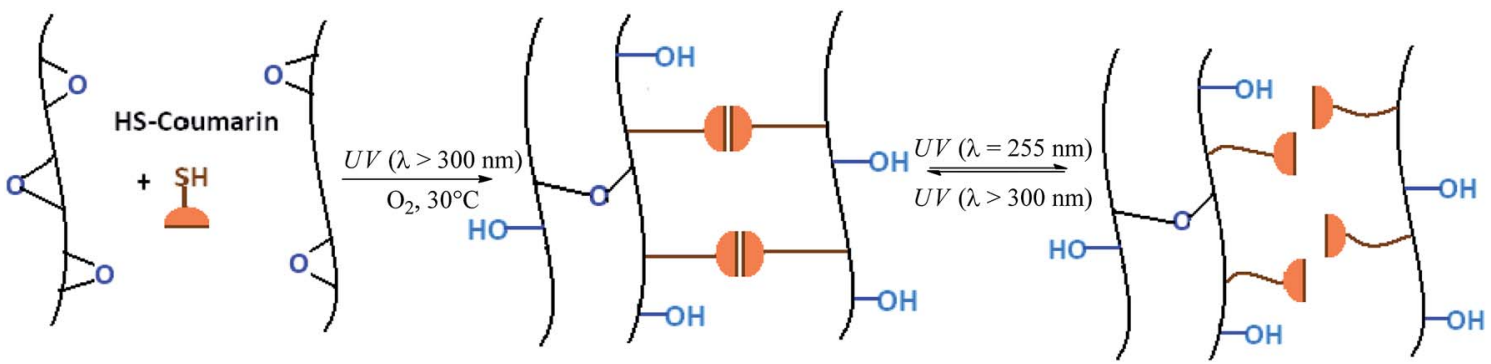

Scheme 1 Coating preparation by photoinitiated thiol-epoxy reaction between epoxidized cottonseed oil and 7-mercapto-4-methyl coumarin under UV and air and its photoinduced self-healing behavior.

purchased from Sigma Aldrich, and 12-dodecanethiol (98\%) obtained from Merck-Schuchardt were used as thiol reactants. Deuterated chloroform, dichloromethane (>99\%) and acetone $(>99 \%)$ were supplied by VWR. All materials were used as received.

The photobase generator (PBG), presented in Scheme 2, was prepared as described in the literature. ${ }^{26}$ 1,5,7-Triazabicyclo [4.4.0]dec-5-ene (TBD, 98\%) and sodium tetraphenylborate $\left(\mathrm{NaBPh}_{4}, 100 \%\right)$ were purchased from Sigma Aldrich. Chlorohydric acid (37\%) was bought from Acros. Isopropylthioxanthone (ITX, >99\%) was kindly supplied by IGM resins and 2,2,6,6-tetramethylpiperidine-1-oxyl (TEMPO, 98\%) was purchased from Acros.

\section{Kinetic study of the photochemical reactions}

Formulations with molar dodecanethiol/epoxy ratios ranging from 0.25 to 1 were prepared in acetone at a $900 \mu \mathrm{g} \mathrm{mL}^{-1}$ concentration. Formulations with molar MMC/epoxy ratios equal to 0.25 and 0.5 were prepared in dichloromethane at the same $900 \mu \mathrm{g} \mathrm{mL}{ }^{-1}$ concentration. A three-component $(0.5 \mathrm{wt} \%$ ITX $+1 \mathrm{wt} \% \mathrm{TBD} \cdot \mathrm{HBPh}_{4}+0.5 \mathrm{wt} \%$ TEMPO) photoinitiating system was added to each formulation. Reagents were stirred during $1 \mathrm{~min}$.

\section{Photo-reversibility study of MMC}

A $900 \mathrm{mg} \mathrm{mL}{ }^{-1}$ solution of MMC in dichloromethane was casted on a quartz plate. The dimerization process was carried out by placing the plate at $10 \mathrm{~cm}$ from a polychromatic light coming from a $\mathrm{Hg}$-Xe lamp (Hamamatsu LC8) fitted with a filter selecting wavelengths between $300 \mathrm{~nm}$ and $450 \mathrm{~nm}$. The incident light intensity was measured at $365 \mathrm{~nm}$ and set to $120 \mathrm{~mW}$ $\mathrm{cm}^{-2}$. For the retro-reaction, a $254 \mathrm{~nm}$ interferential filter was used and the intensity of the monochromatic light was kept at $0.6 \mathrm{~mW} \mathrm{~cm}{ }^{-2}$.

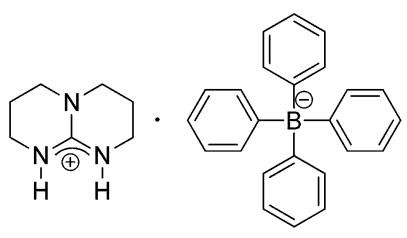

Scheme 2 Chemical structure of the photolatent base TBD $\cdot \mathrm{HBPh}_{4}$.

\section{Coatings preparation}

A 0.25 molar thiol/epoxy ratio and 2 wt\% PBG were used. Formulation was prepared at a $90 \mu \mathrm{g} \mathrm{mL} \mathrm{m}^{-1}$ concentration in dichloromethane, stirred during $1 \mathrm{~min}$ and then applied onto a glass substrate. The film was then irradiated under air for 20 min by a filtered polychromatic light coming from a $\mathrm{Hg}-\mathrm{Xe}$ lamp (Hamamatsu LC8). The emission spectrum was restricted to the range $300-450 \mathrm{~nm}$ and the incident light intensity measured at $365 \mathrm{~nm}$ was $120 \mathrm{~mW} \mathrm{~cm}^{-2}$.

\section{Analytical techniques}

${ }^{1} \mathrm{H}$ NMR analyses were carried out on a Bruker $300 \mathrm{MHz}$ spectrometer at room temperature. The chemical shifts are given in ppm relative to trimethylsilane (TMS) used as external reference. Samples were prepared as follow: $10 \mathrm{mg}$ of product in 0.4 $\mathrm{mL}$ of $\mathrm{CDCl}_{3}$.

Fourier Transform Infra-Red (FTIR) spectra were performed on a Vertex 70 Bruker spectrometer equipped with an ATR accessory (ATR MK II Golden Gate Specac, Eurolabo) at $30^{\circ} \mathrm{C}$. A drop of the sample was applied to the horizontal ATR crystal and then covered or not by a polyethylene film before irradiation. The UV light coming from a polychromatic $\mathrm{Hg}-\mathrm{Xe}$ lamp (Hamamatsu LC8) was applied while IR spectra were recorded versus time. Epoxy group, thiol function and coumarin's double bond consumption was determined by following the absorbance of the peaks at $823 \mathrm{~cm}^{-1}$ (deformation band of oxirane cycle), $2550 \mathrm{~cm}^{-1}$ (S-H stretching band) and $3100 \mathrm{~cm}^{-1}$ (symmetric stretching band of cis configuration alkene), respectively. The formation of thioether bond and hydroxyl group was monitored by following the absorbance of the peaks at $900 \mathrm{~cm}^{-1}$ (C-S deformation band) and $3500 \mathrm{~cm}^{-1}(\mathrm{OH}$ stretching band), respectively. Reaction kinetics was plotted using the absorbance variation of the different peaks: $\Delta A=A_{0}-A_{t}$ where $A_{0}$ and $A_{t}$ refer to the absorbance of the corresponding peak at $t=0$ and $t$, respectively.

UV spectra were performed using a Cary 100 Varian UVvisible spectrometer. The formulation was spread out on a quartz window and analyzed between 200 and $400 \mathrm{~nm}$.

Size Exclusion Chromatography (SEC) analyses were carried out with a Varian 50 system and equipped with a pre-column (Polymer Laboratories, PL gel $5 \mu \mathrm{m}$ Guard, $50 \times 7.5 \mathrm{~mm}$ ) and two columns (Polymer Laboratories, 2 PL gel $5 \mu \mathrm{m}$ MIXED-D columns, $2 \times 300 \times 7.5 \mathrm{~mm}$ ) and a refractive index detector 
(differential refractometer, sensibility: $2.5 \times 10^{7}$ RIU per $\mathrm{mV}$, wavelength $880 \pm 30 \mathrm{~nm}$ ). Dichloromethane was used as eluent with a $1 \mathrm{~mL} \min ^{-1}$ flow at $35{ }^{\circ} \mathrm{C}$. The system was calibrated using polymethylmethacrylate (PMMA) standards. Samples were analysed with an internal standard (PMMA, $\overline{M_{\mathrm{n}}}=29600$ $\mathrm{g} \mathrm{mol}{ }^{-1}, 20 \%$ of the sample mass $)$ after filtration $(0.45 \mu \mathrm{m})$.

Thermogravimetric analyses (TGA) were performed on a TGA Q500 device from TA Instruments (platinum pan, $10 \mathrm{~mL} \mathrm{~min}{ }^{-1}$ constant flow of nitrogen, temperature range from $30{ }^{\circ} \mathrm{C}$ to 600 ${ }^{\circ} \mathrm{C}, 10{ }^{\circ} \mathrm{C} \min ^{-1}$ heating rate).

Differential Scanning Calorimetry (DSC) analyses were performed on a DSC Q2000 device from TA Instruments (aluminum pan, $10 \mathrm{~mL} \min ^{-1}$ constant flow of nitrogen, temperature range from $-80{ }^{\circ} \mathrm{C}$ to $120{ }^{\circ} \mathrm{C}, 10{ }^{\circ} \mathrm{C} \mathrm{min}{ }^{-1}$ heating rate).

Film extraction was carried out as follows: $50 \mathrm{mg}$ of sample were added into a $100 \mathrm{~mL}$ flask and extracted with $70 \mathrm{~mL}$ dichloromethane. The extraction was carried out for $24 \mathrm{~h}$ in cellulose vessels, under atmospheric pressure and at the temperature of $60{ }^{\circ} \mathrm{C}$ (reflux). The extraction yield was determined from weighing of the residue:

$$
\text { Extraction yield }(\%)=\frac{\text { residue mass }}{\text { sample mass }} \times 100
$$

The extraction liquids were collected, concentrated in a rotary evaporator at $40{ }^{\circ} \mathrm{C}$ and then analyzed by SEC according to the aforementioned protocol.

Surface energy of the materials was evaluated using a Digidrop Goniometer (GBX, France). Static contact angles were measured at the equilibrium time with three liquids: water, glycerol and diiodomethane. Five measurements were performed for each sample surface to calculate the average contact angle and its standard deviation. According to the OwensWendt relationship, the dispersive $\gamma^{\mathrm{d}}$ and polar $\gamma^{\mathrm{p}}$ components and the surface energies $\left(\gamma_{\mathrm{s}}=\gamma^{\mathrm{d}}+\gamma^{\mathrm{p}}\right)$ of the samples were determined. Surface polarities $(P)$ of the materials were also calculated using equation:

$$
P(\%)=\frac{\gamma^{\mathrm{p}}}{\gamma_{\mathrm{s}}} \times 100
$$

Film hardness was investigated using a König pendulum SP0500 (Labomat Essor). Three measurements were performed for each sample to calculate the average König hardness and its standard deviation.

\section{Results and discussion}

Thiol-ene addition is the usual pathway for the functionalization of unsaturated vegetable oils by thiol reagents. ${ }^{27,28}$ Nevertheless, this pathway presents many disadvantages such as instability of the reaction medium under storage and numerous side reactions. ${ }^{29}$ For these reasons, the thiol-epoxy addition was preferred to the thiol-ene one in this work. The fatty raw material was a cottonseed oil having 4 ethylenic double bonds per triglyceride molecule in its native form (determined by ${ }^{1} \mathrm{H}$
NMR analysis).$^{30}$ A peroxidative process was used as mentioned in the experimental part to prepare epoxidized oil. ${ }^{1} \mathrm{H}$ NMR analysis of the ECO showed an average number of 4 epoxy groups per triglyceride molecule (the presence of residual ethylenic double bond was estimated to 7\%). 7-Mercapto-4methyl-coumarin (MMC) was chosen as thiol monomer and for its photo-reversible cyclization.

According to literature, thiol-epoxy addition can be initiated by tertiary amines. Two initiation pathways can occur as a function of the $\mathrm{p} K_{\mathrm{a}}$ of initiator and thiol group. ${ }^{31}$ In the presence of a good hydrogen donor (e.g. thiol with low $\mathrm{p} K_{\mathrm{a}}$ ), base initiation is observed. In this case, the base can abstract the thiol hydrogen to form a thiolate anion, which then opens the oxyrane group. In the opposite case, a nucleophilic initiation rather occurs. Thus, an alcoolate group is formed by addition of the amine onto the epoxy function before addition on the thiol function. An anionic epoxy homopolymerization can then ensue when there is no more hydrogen donor in the reaction medium. ${ }^{\mathbf{1 4}}$

In this work, the photochemical initiation way was privileged. For that, a photobase generator composed of a latent triamine (TBD $\cdot \mathrm{HBPh}_{4}$ ) and a photosensitizer (ITX) in 1/0.5 wt\% proportions, respectively, was used. As the basicity of the PGB is very strong $\left(\mathrm{p} K_{\mathrm{a}}=15.2\right)^{26}$ and far greater than the acidity of the thiol coumarin derivative $\left(\mathrm{p} K_{\mathrm{a}}=7-8\right),{ }^{17}$ the initiation step can be considered as a base pathway (Scheme 3). It was also noticed that $0.5 \mathrm{wt} \%$ of TEMPO was added as radical inhibitor.

\section{Model kinetic study of the thiol-epoxy reaction}

In order to determine the optimal composition of the thiol/oil formulation, a kinetic study of the thiol/epoxy reaction was first carried out. To model the MMC behavior, dodecanethiol (DT, $\left.\mathrm{p} K_{\mathrm{a}}=10.2\right)^{17}$ was chosen due to its good solubility into oil. Reaction kinetics was monitored by real time infrared spectroscopy at $30{ }^{\circ} \mathrm{C}$. A polychromatic UV light with an incident intensity of $120 \mathrm{~mW} \mathrm{~cm} \mathrm{~cm}^{-2}$ (measured at $365 \mathrm{~nm}$ ) was used. Although absorption bands corresponding to $\mathrm{S}-\mathrm{H}$ bond at 2550 $\mathrm{cm}^{-1}$ and oxirane group at $823 \mathrm{~cm}^{-1}$ can be observed on IR spectra, the low intensity of the first band and the poor resolution of the second one did not allow calculating conversions accurately as shown on Fig. 1. Hence, only the epoxy absorbance decrease kinetics was presented here. Besides, the absorbance increase of $\mathrm{C}-\mathrm{S}$ bond and $\mathrm{OH}$ group was also followed.

The influence of oxygen onto thiol-epoxy addition was firstly investigated. A stoichiometric thiol/epoxy formulation was prepared and spread out on the ATR crystal. Samples were then irradiated under air or in laminated layer in the presence of 2 wt $\%$ of PBG. Fig. 2 shows that the absorbance variation kinetics for the epoxy group was greater under air. Hu et al. have recently shown that the UV exposure of aromatic amines similar to TBD in the presence of photosensitizer could induce numerous

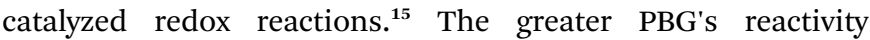
observed under air and UV radiation could possibly be explained by such reactions that could induce a faster base releasing. The following experiments were then carried out under air. 


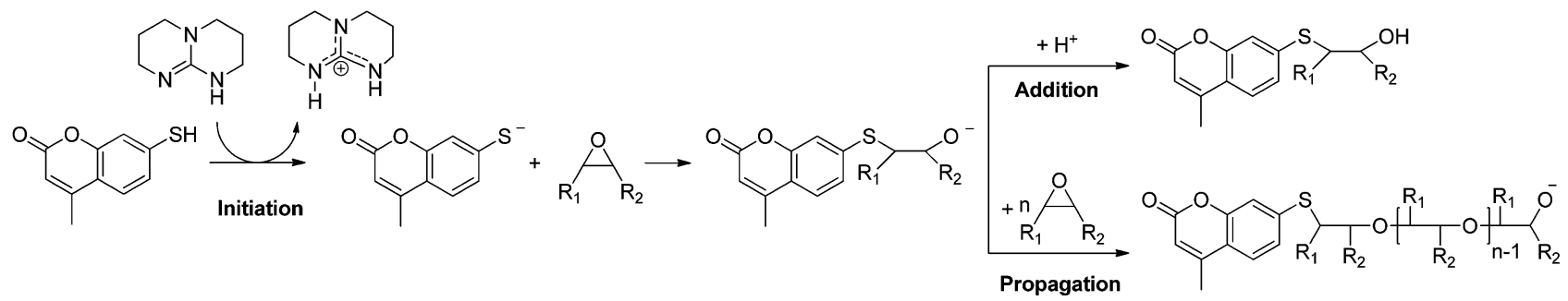

Scheme 3 Base initiated mechanism of the thiol-epoxy reaction.

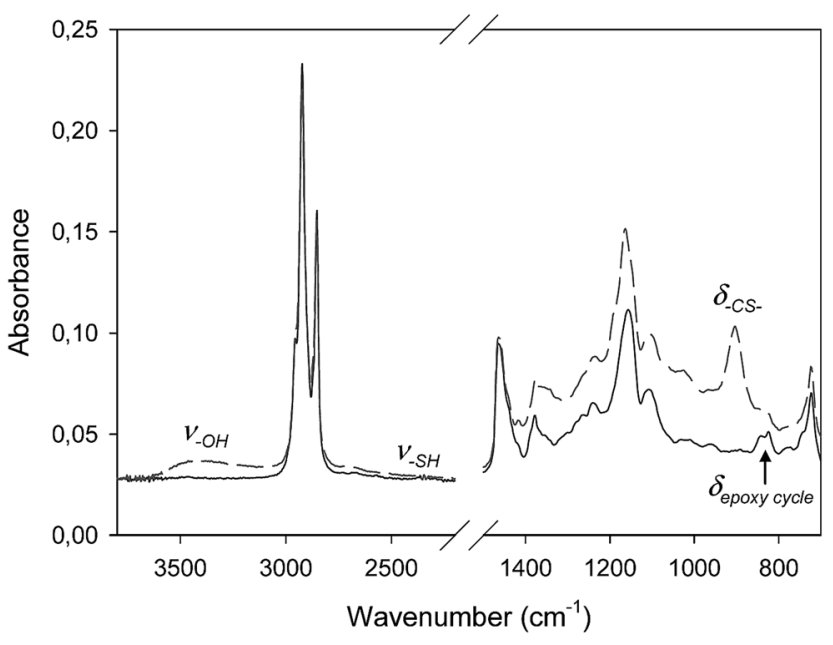

Fig. 1 IR spectra of epoxidized cottonseed oil + dodecanethiol (molar ratio $1: 1$ in functions) $+2 \mathrm{wt} \% \mathrm{PBG}$ at $t=0$ (solid line) and after $20 \mathrm{~min}$ UV irradiation (dashed line) $-T=30^{\circ} \mathrm{C}$, polychromatic irradiation $\left(I_{0}=\right.$ $120 \mathrm{~mW} \mathrm{~cm}^{-2}$ at $365 \mathrm{~nm}$ ).

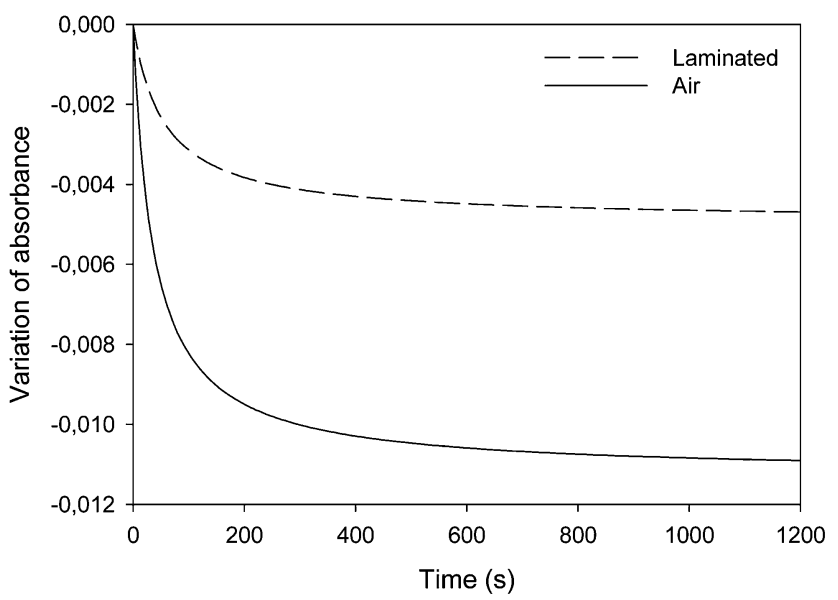

Fig. 2 Influence of oxygen on the epoxy absorbance variation kinetics of epoxidized cottonseed oil + dodecanethiol (molar ratio 1:1 in functions $)+2 \mathrm{wt} \% \mathrm{PBG}-T=30{ }^{\circ} \mathrm{C}$, polychromatic irradiation $\left(/_{0}=\right.$ $120 \mathrm{~mW} \mathrm{~cm}^{-2}$ at $365 \mathrm{~nm}$ ).

The PBG amount was then varied from 0 to $3 \mathrm{wt} \%$. As expected, the results showed that the consumption of epoxy functions (Fig. 3a) as well as the formation of $\mathrm{C}-\mathrm{S}$ bond and $\mathrm{OH}$ group (Fig. 3b and c, respectively) was more important as the quantity of PBG increased. Nonetheless, this trend seems to diminish for higher PGB concentrations, probably due to the limited solubility of PBG into oil. We therefore decided to keep a PBG concentration of $2 \mathrm{wt} \%$ in the following experiments. It is also noteworthy that no $\mathrm{C}-\mathrm{S}$ bond appeared in the absence of PBG, indicating the requirement of the photocatalytic system to initiate the reaction confirming the stability of the mixture under storage. However, we also observed a slight formation of epoxy and $\mathrm{OH}$ groups in this case. This could be explained by the presence of hydroperoxydes in the vegetable oil, which are well known to generate ${ }^{1} \mathrm{O}_{2}$ under UV radiation. ${ }^{32,33}$ This oxygen molecule could then oxidize the residual ethylenic double bonds of the epoxidized cottonseed oil.

Last, the influence of thiol quantity (from 0 to 1 equivalent $\mathrm{SH}$ relative to epoxy function) was studied. As expected, Fig. 4 shows that the epoxy consumption (Fig. 4a) as well as the C-S bond and $\mathrm{OH}$ bond formation (Fig. $4 \mathrm{~b}$ and c, respectively) progressed more quickly when the quantity of thiol was more important. The soluble fractions of the formulations before and after irradiation were analyzed by SEC with an internal standard after filtration. Results showed that higher concentrations of thiol increased the dimerization of thiol monomers $(11 \%$ for 0.5 eq. MMC against $6 \%$ for 0.25 eq. MMC). As a result, thiol-epoxy addition was much lower. We therefore decided to use a molar thiol/epoxy ratio of 0.25 in the following study.

\section{Kinetic study of the MMC photo-reversibility}

The photo-reversibility of the (non grafted) MMC at solid state was firstly studied. MMC was first solubilized in dichloromethane and spread out on a quartz plate. After solvent evaporation, MMC was first irradiated by a polychromatic light (300 $<\lambda<450 \mathrm{~nm}-I_{0}=120 \mathrm{~mW} \mathrm{~cm}^{-2}$ at $365 \mathrm{~nm}$ ) under air for 90 min and then by a monochromatic light $\left(\lambda=255 \mathrm{~nm}-I_{0}=0.6\right.$ $\mathrm{mW} \mathrm{cm}{ }^{-2}$ ) under air for $120 \mathrm{~min}$. The photo-reversibility of the coumarin moiety was monitored by UV-visible spectrophotometry (Fig. 5). Indeed, the coumarin group initially exhibited an absorbance maximum at $\lambda_{\max }=330-340 \mathrm{~nm} .{ }^{34}$ When exposed to the UV-B/C radiation, the $[2+2]$ cycloaddition occurred and led to a decrease of this absorbance maximum until a plateau was reached after $60 \mathrm{~min}$ irradiation (Fig. 5a). By exposure to the $255 \mathrm{~nm}$ radiation, an absorbance increase was observed (Fig. 5b), indicating the photocleavage of the cyclobutane and the reformation of double bonds (Scheme 4a). In the course of the second irradiation cycle, the photo-reversibility of MMC was again observed. However, we could remark the absorbance 

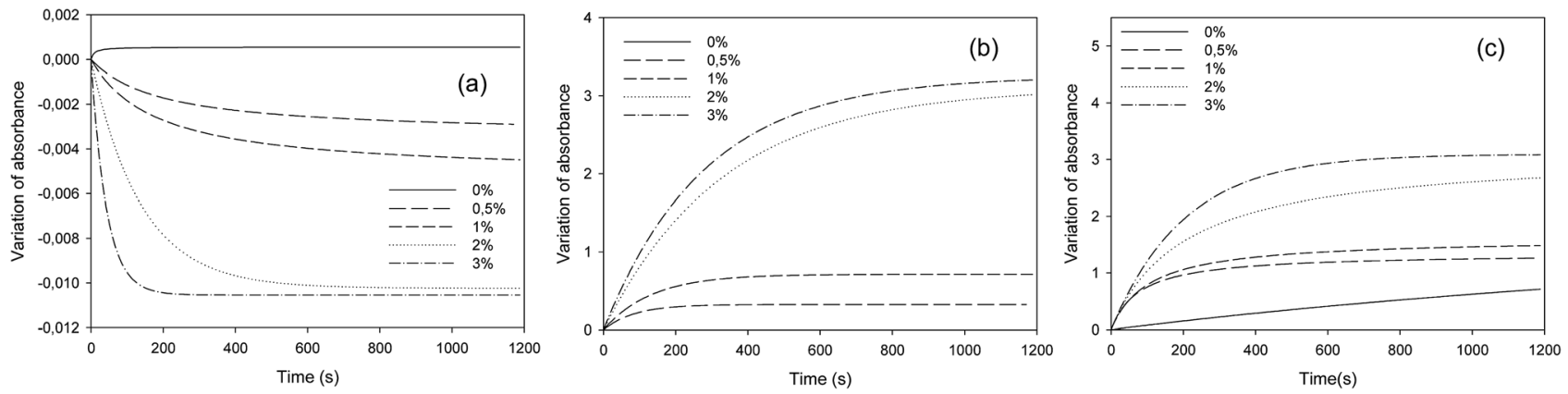

Fig. 3 Influence of PGB quantity on the absorbance variation kinetics of (a) epoxy function, (b) C-S bond, (c) - OH group for a mixture composed of epoxidized cottonseed oil + dodecanethiol (molar ratio $1: 1$ in functions) +2 wt $\%$ PBG $-T=30{ }^{\circ} \mathrm{C}$, polychromatic irradiation $(/ 0=120$ $\mathrm{mW} \mathrm{cm}{ }^{-2}$ at $\left.365 \mathrm{~nm}\right)$, under air.
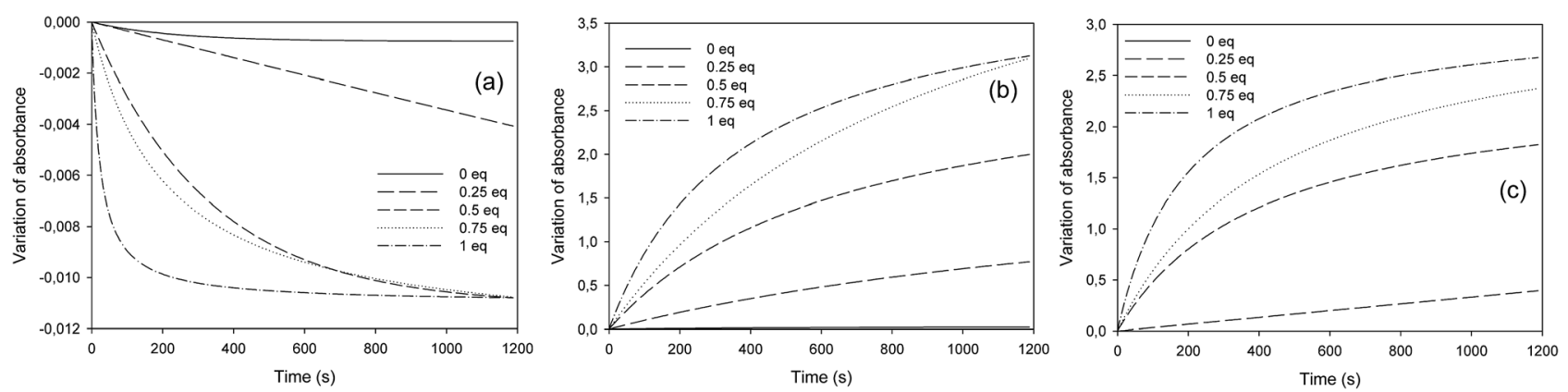

Fig. 4 Influence of thiol quantity on the absorbance variation kinetics of (a) epoxy function, (b) C-S bond, (c) -OH group for a mixture composed of epoxidized cottonseed oil $+2 \mathrm{wt} \% \mathrm{PBG}-T=30^{\circ} \mathrm{C}$, polychromatic irradiation $\left(I_{0}=120 \mathrm{~mW} \mathrm{~cm}^{-2}\right.$ at $\left.365 \mathrm{~nm}\right)$, under air.
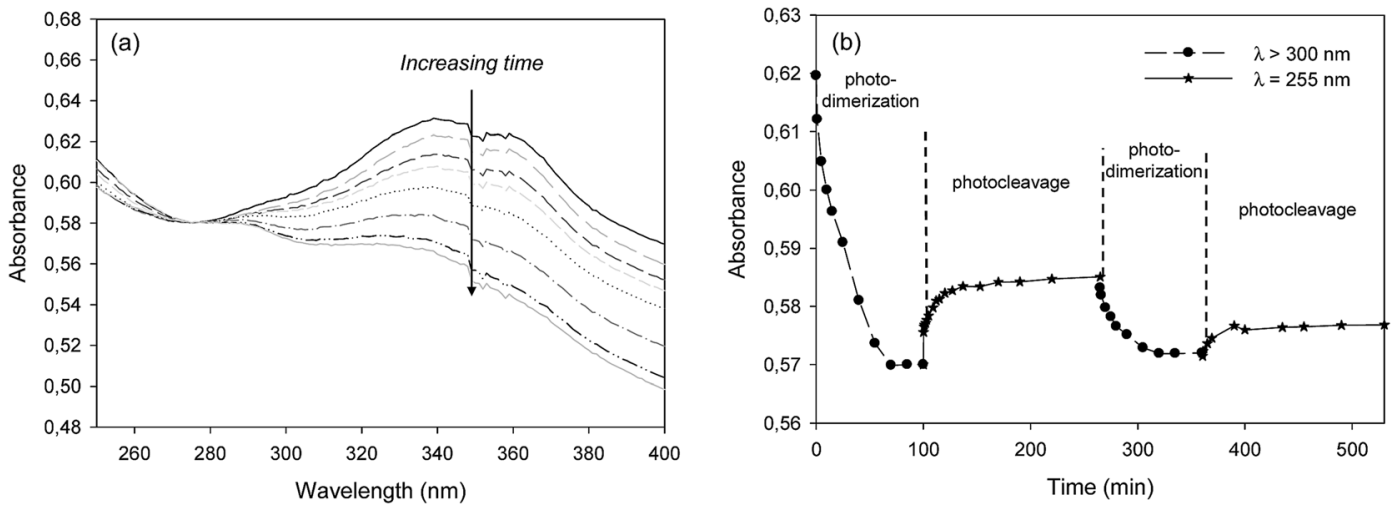

Fig. 5 Evolution of the MMC peak absorbance at $330 \mathrm{~nm}$ during (a) the first irradiation at $300<\lambda<450 \mathrm{~nm}\left(I_{0}=120 \mathrm{~mW} \mathrm{~cm}^{-2}\right.$ at $\left.365 \mathrm{~nm}\right)$ and (b) several irradiation cycles (polychromatic irradiation at $300<\lambda<450 \mathrm{~nm}\left(I_{0}=120 \mathrm{~mW} \mathrm{~cm}{ }^{-2}\right.$ at $\left.365 \mathrm{~nm}\right)$ and monochromatic irradiation at $\lambda=255$ $\left.\mathrm{nm}\left(I_{0}=0.6 \mathrm{~mW} \mathrm{~cm}^{-2}\right)\right)$ at $T=30{ }^{\circ} \mathrm{C}$ and under air.

variation amplitude was lower than after the first irradiation cycle (Fig. 5b).

A further kinetic monitoring of the photochemical reaction by FTIR spectroscopy well highlighted not only the variation of the double bonds band absorbance during the different irradiation steps, but also the appearance of the $\mathrm{C}-\mathrm{S}$ absorption band at $900 \mathrm{~cm}^{-1}$. The formation of $\mathrm{C}-\mathrm{S}$ bond could only be explained by a thiol-ene reaction (Scheme $4 \mathrm{~b}$ ) between the thiol group of MMC and its double bond via a radical process, which is favoured by the high MMC concentration. This reaction could probably explain the attenuation of the photo-reversibility after several irradiation cycles.

Once grafted onto the epoxidized cottonseed oil and the formulation photocured, the photo-reversibility of the MMC was secondly studied at solid state. Formulation composed of a 0.25 molar thiol/epoxy ratio and $2 \mathrm{wt} \%$ PBG was spread out on quartz plate and exposed to similar irradiation conditions as previously described. Fig. 6 showed that the mixture before 
(a)<smiles>Cc1cc(=O)oc2cc(S)ccc12</smiles>

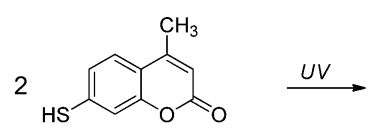

Scheme 4 Possible MMC's dimerization pathways under UV radiation: (a) $[2+2]$ cycloaddition (major); (b) thiol-ene addition (minor).

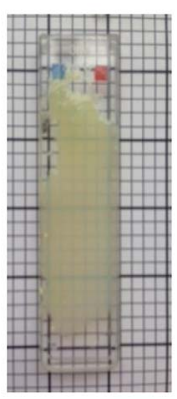

Before irradiation

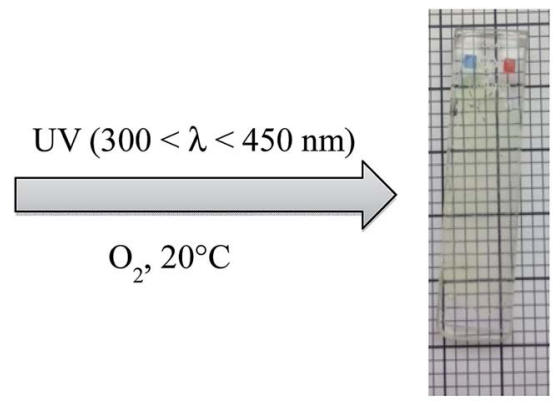

After irradiation

Fig. 6 Films composed of cottonseed oil + dodecanethiol (molar ratio $1: 0.25$ in functions) $+2 w t \%$ PBG before and after irradiation at $300<$ $\lambda<450 \mathrm{~nm}\left(I_{0}=120 \mathrm{~mW} \mathrm{~cm}^{-2}\right.$ at $\left.365 \mathrm{~nm}\right)$.

irradiation was opaque and non homogeneous whereas the coating was entirely transparent and homogeneous after irradiation, meaning a good incorporation of MMC by grafting onto oil. It is noteworthy that the reaction was carried out in the presence of TEMPO. Hence, the MMC grafting via the thiolepoxy reaction rather occurred than the radical thiol-ene addition.

As in the previous study, the photo-reversibility of the grafted MMC was monitored by FTIR and UV-visible spectroscopies. Fig. 7 presents the kinetics of MMC's double bond and $\mathrm{SH}$ function consumption and of $\mathrm{C}-\mathrm{S}$ bond and $-\mathrm{OH}$ group formation.

\section{Characterization of materials}

10-20 $\mu \mathrm{m}$ thick films were elaborated according to the optimal conditions previously determined. A formulation containing 0.25 eq. MMC relative to fatty epoxy group and $2 \mathrm{wt} \%$ of PGB was then irradiated at $30{ }^{\circ} \mathrm{C}$ with a polychromatic light from 300 to $450 \mathrm{~nm}$ and an incident light intensity $I_{0}=120 \mathrm{~mW} \mathrm{~cm}{ }^{-2}$ measured at $365 \mathrm{~nm}$. The physicochemical properties of the films were then characterized.

In a general way, the photocured films are transparent and flexible. They do not release any thiol smell and are not sticky after irradiation. FTIR analyses of the photocured films showed a complete consumption of both MMC's double bond and $\mathrm{SH}$

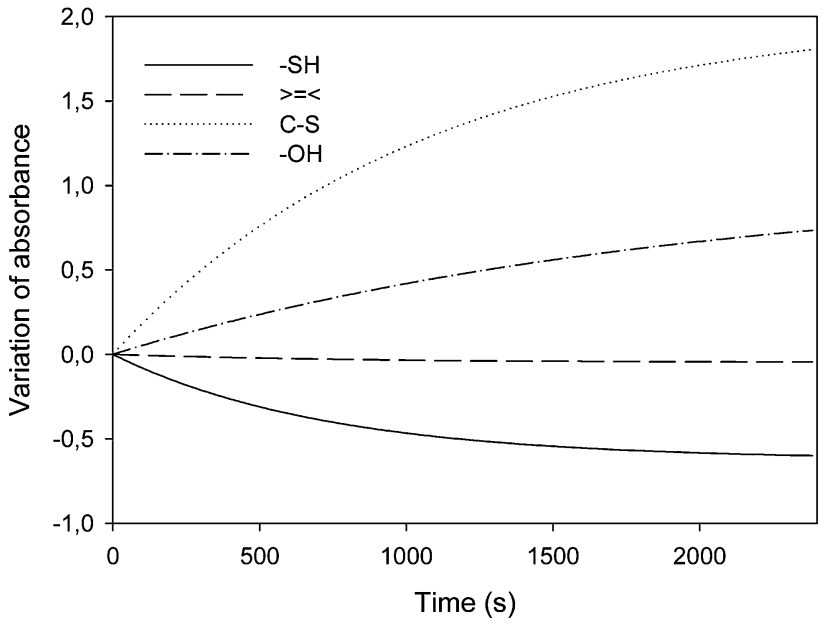

Fig. 7 Absorbance variation kinetics of thiol, MMC's double bond, C-S bond and $\mathrm{OH}$ group for a mixture composed of epoxidized cottonseed oil + MMC (molar ratio 1: 0.25 in functions) $+2 w t \% P B G-T=30$ ${ }^{\circ} \mathrm{C}$, polychromatic irradiation at $300<\lambda<450 \mathrm{~nm}\left(I_{0}=120 \mathrm{~mW} \mathrm{~cm}^{-2}\right.$ at $365 \mathrm{~nm}$ ), under air.

group as well as the consumption of epoxy functions and the formation of $\mathrm{OH}$ groups. Furthermore, extraction was carried out on the obtained films and extractables were analyzed by both ${ }^{1} \mathrm{H}$ NMR and SEC in the presence of an internal standard. The soluble fraction was estimated to $65 \%$ and was mainly composed of $80 \mathrm{wt} \%$ of epoxidized triglycerides $\left(\overline{M_{\mathrm{n}}}=990\right.$ $\mathrm{g} \mathrm{mol}^{-1}-D=1.03$ ) and $16 \mathrm{wt} \%$ of a mixture of thiol-epoxy adducts and/or triglyceride oligomers with low molecular weight $\left(\overline{M_{\mathrm{n}}}=2700-3200 \mathrm{~g} \mathrm{~mol}^{-1}-D=1.08\right)$. Only $2 \mathrm{wt} \%$ of MMC and $2 \mathrm{wt} \%$ of MMC dimers were detected by SEC whereas NMR ${ }^{1} \mathrm{H}$ analysis showed no residual thiol after irradiation. All these results showed that MMC was well grafted onto epoxidized triglycerides and dimerized.

TGA and DSC analyses of the photocured films before and after extraction as well as of the epoxidized cottonseed oil were performed. Fig. 8 highlighted that the films' degradation could be divided into two parts, which were more pronounced for the washed film. The first part could be attributed to the degradation of free triglycerides and/or its oligomers till $300{ }^{\circ} \mathrm{C},{ }^{35}$ whereas the second one could be related to the degradation of aromatic structures. Furthermore, it could be noticed that the degradation temperature of the films was lower than the epoxidized cottonseed oil one. This could be explained by the thermally activated cleavage of $\mathrm{C}-\mathrm{S}$ or $\mathrm{S}-\mathrm{S}$ bond, which exhibit lower bond energy than $\mathrm{C}-\mathrm{C}$ and $\mathrm{C}-\mathrm{O}$ ones. ${ }^{36}$ Besides, according to DSC analysis, the film after extraction exhibited a glass transition temperature of $-13{ }^{\circ} \mathrm{C}$. This temperature was higher than the one measured before extraction, free low molecular weight molecules acting as plasticizer. Despite of this low $T_{\mathrm{g}}$ value, the non washed film exhibited a relatively high König hardness estimated to $102 \pm 18 \mathrm{~s}$. Last, the polarity of the photocured film was estimated to $15 \pm 4 \%$.

To finish, the photo-reversibility properties of the film were evaluated. Several irradiation cycles were applied. Each cycle was consisted of $180 \mathrm{~min}$ irradiation at $300<\lambda<450 \mathrm{~nm}\left(I_{0}=\right.$ 


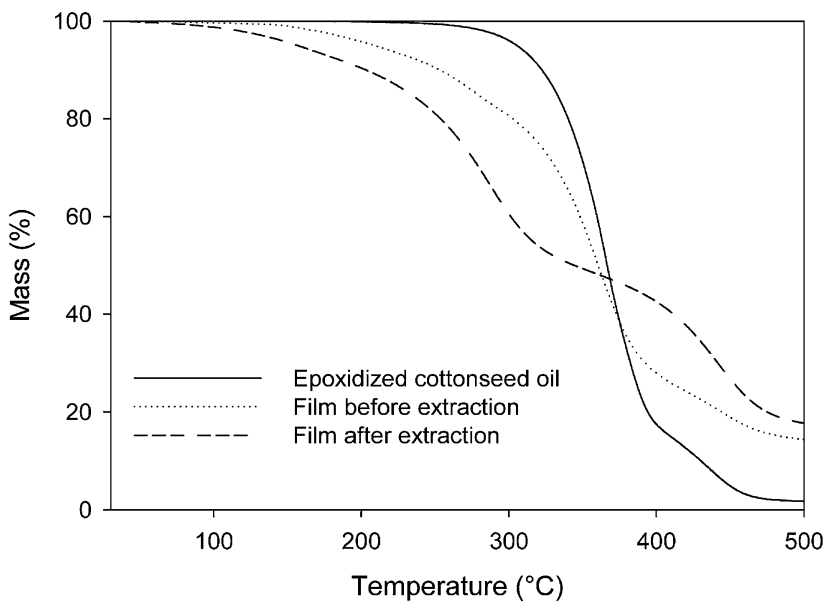

Fig. 8 Thermal degradation curves for epoxidized cottonseed oil and photocured films before and after extraction.

$120 \mathrm{~mW} \mathrm{~cm}^{-2}$ at $\left.365 \mathrm{~nm}\right)$ and $8 \mathrm{~h}$ irradiation at $\lambda=255 \mathrm{~nm}\left(I_{0}=\right.$ $0.6 \mathrm{~mW} \mathrm{~cm}^{-2}$ ). The monitoring of the MMC photo-reversibility by UV spectroscopy (Fig. 9) showed a decrease of the absorbance maximum at $330 \mathrm{~nm}$ from 3.4 to 1.4 during the irradiation step at $300<\lambda<450 \mathrm{~nm}$, indicating the MMC dimerization. The photodimerized film was then subjected to the radiation at $\lambda=$ $255 \mathrm{~nm}$. With the progress of the retro-dimerization, the film absorbance at $330 \mathrm{~nm}$ slowly increased from 1.4 to 2.0 after $8 \mathrm{~h}$ irradiation. This great reaction time and the limited recovery could be explained by the very weak irradiation intensity, which was delivered by the $\mathrm{Hg}$-Xe lamp at $255 \mathrm{~nm}$. A new irradiation at $300<\lambda<450 \mathrm{~nm}$ led again to a decrease of the maximum absorbance of the MMC consecutive to its dimerization. The photo-reversibility of the film was therefore confirmed.

The self-healability of the system was also quantified by pendulum hardness measurement. The König hardness of the film was determined after each irradiation step and reported in Fig. 10 as a function of the irradiation cycles number. Results

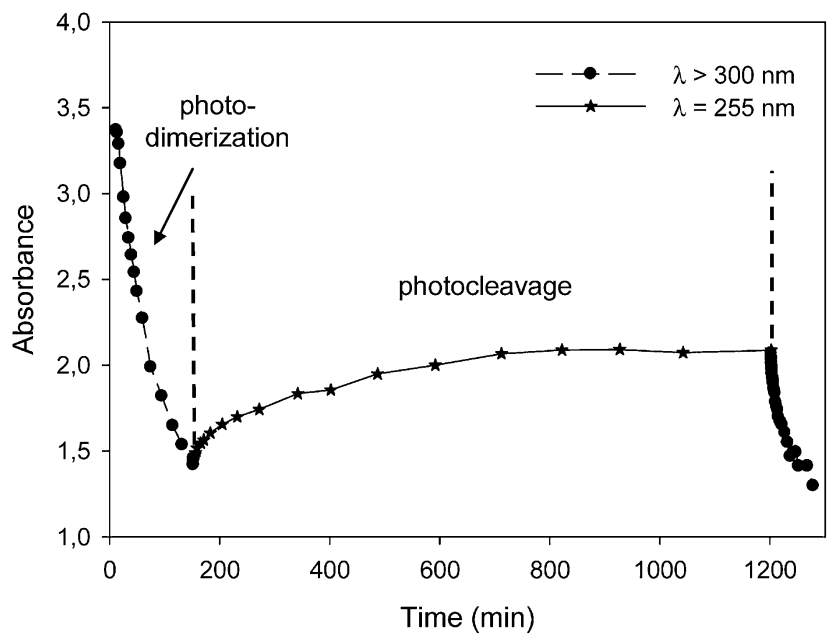

Fig. 9 Evolution of the grafted MMC peak absorbance at $330 \mathrm{~nm}$ during several irradiation cycles.

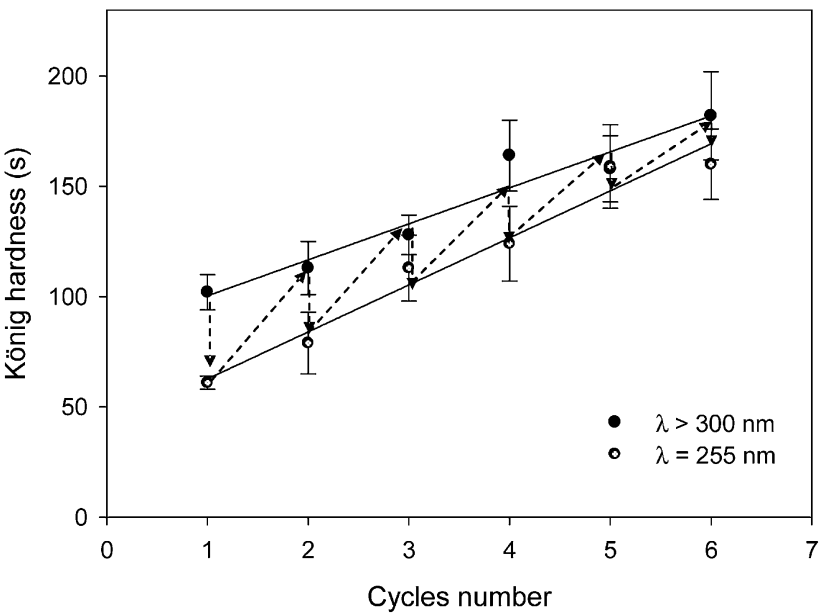

Fig. 10 Evolution of the König hardness of the photocured film during several irradiation cycles.

highlighted several interesting points. First, the decrease of the film hardness after irradiation at $\lambda=255 \mathrm{~nm}$ could be considered as the best evidence for the photocleavage of the grafted MMC dimer into the grafted MMC monomer. Second, hardness can be recovered after irradiation at $300<\lambda<450 \mathrm{~nm}$, confirming again the photo-reversibility of the dimerization. However, it is noteworthy that the restored hardness was higher than the one measured in the previous irradiation cycle. The significant increase of the film hardness in the course of the irradiation steps could be explained by the homopolymerization of epoxy groups that occurred and/or continued under all irradiation conditions, as confirmed by FTIR. The resulting higher crosslinking density could also induce a reducing of the coumarin moieties mobility and explain the limitation of the reversibility recovery as observed in Fig. 9.

\section{Conclusions}

In this study, a novel biosourced photo-healable material was prepared by photoinitiated thiol-epoxy chemistry. This new coating is constituted of $80 \mathrm{wt} \%$ of epoxidized cottonseed oil and $20 \mathrm{wt} \%$ of 7 -mercapto-4-methyl coumarin. The reaction could be performed under ambient conditions (room temperature and air). The MMC's photografting onto epoxidized vegetable oil via the thiol-epoxy addition and its photodimerization were clearly confirmed. Furthermore, the ring opening polymerization of fatty epoxy groups was also observed in the presence of PBG. This side reaction enhanced the crosslinking density of the final coating in the course of the photo-healing steps. At last, it could be noteworthy that this is the first photoinduced self-healing material prepared by photoinitiated thiol-epoxy chemistry from vegetable oil derivatives.

\section{Acknowledgements}

This work has been supported by the French Government (MESR fellowship). The authors are grateful to S. Leveneur and 
J.-L. Zheng of the Laboratoire de Sécurité des Risques Chimiques (LSPC, INSA de Rouen, France) for the epoxidized cottonseed oil preparation.

\section{Notes and references}

1 O. Türünç and M. A. R. Meier, Green Chem., 2011, 13, 314.

2 M. Desroches, S. Caillol, V. Lapinte, R. Auvergne and B. Boutevin, Macromolecules, 2011, 44, 2489.

3 A. Corneille, V. Froidevaux, C. Negrell, S. Caillol and B. Boutevin, Polymer, 2014, 55, 5561.

4 C. Lluch, J. C. Ronda, M. Galia, G. Lligadas and V. Cadiz, Biomacromolecules, 2010, 11, 1646.

5 G. L. Schneberger, Adhesives in Manufacturing, Marcel Dekker Inc, 1983, vol. 11.

6 A. Brändle and A. Khan, Polym. Chem., 2012, 3, 3224.

7 S. Binder, I. Gadwal, A. Bielmann and A. Khan, J. Polym. Sci., Part A: Polym. Chem., 2014, 52, 2040.

8 K. Arimitsu and R. Endo, Chem. Mater., 2013, 25, 4461.

9 C. F. Carlberg, A. Vastesson, Y. Liu, W. van der Wijingaart, M. Johansson and T. Haraldsson, J. Polym. Sci., Part A: Polym. Chem., 2014, 52, 2604.

10 J. A. Carioscia, J. W. Stansbury and C. N. Bowman, Polymer, 2007, 48, 1526.

11 S. Katogi and M. Yusa, J. Polym. Sci., Part A: Polym. Chem., 2011, 40, 4045.

12 C. M. Seubert and M. E. Nicols, J. Coat. Technol. Res., 2010, 7, 615.

13 D. P. Nair, M. Podgorski, S. Chantani, T. Gong, W. Xi, C. R. Fenoli and C. N. Bowman, Chem. Mater., 2014, 26, 724.

14 J. Lalevée, M. A. Tehfe, F. Morlet-Savary, B. Graff, X. Allonas and J. P. Fouassier, Prog. Org. Coat., 2011, 70, 23.

15 J. Hu, J. Wang, T. H. Nguyen and N. Zheng, Beilstein J. Org. Chem., 2013, 9, 1977.

16 J. A. Carioscia, J. W. Stansbury and C. N. Bowman, Polymer, 2007, 48, 1526.

17 S. Banerjee, R. Tripathy, D. Cozzens, T. Nagy, S. Keki, M. Zsuga and R. Faust, ACS Appl. Mater. Interfaces, 2015, 7, 2064.
18 B. Kiskan and Y. Yagci, J. Polym. Sci., Part A: Polym. Chem., 2014, 52, 2911.

19 P. Froimowicz, H. Frey and K. Landfester, Macromol. Rapid Commun., 2011, 32, 468.

20 Y. Amamoto, J. Kamada, H. Otsuka, A. Takahara and K. Matyjaszewski, Angew. Chem., Int. Ed., 2011, 50, 1660.

21 M. Nagata and Y. Yamamoto, React. Funct. Polym., 2008, 68, 915.

22 Y. Chujo, K. Sada and T. Saegusa, Macromolecules, 1990, 23, 2693.

23 J. Ling, M. Z. Rong and M. Q. Zhang, Polymer, 2012, 53, 2691.

24 E. Sato, S. Nagai and A. Matsumoto, Prog. Org. Coat., 2013, 76, 1747.

25 J. L. Zheng, J. Wärna, F. Burel, T. Salmi, B. Taouk and S. Leveneur, AIChE J., 2016, 62, 726.

26 X. Sun, J. P. Gao and Z. Y. Wang, J. Am. Chem. Soc., 2008, 130, 8130.

27 M. Desroches, S. Caillol, V. Lapinte, R. Auvergne and B. Boutevin, Macromolecules, 2011, 44, 2489.

28 M. Ionescu, D. Radojcic, X. Wan, Z. S. Petrovic and T. A. Upshaw, Eur. Polym. J., 2015, 67, 439.

29 C. N. Bowman and C. E. Hoyle, Angew. Chem., Int. Ed., 2010, 49, 1540.

30 O. Zovi, L. Lecamp, C. Loutelier-Bourhis, C. M. Lange and C. Bunel, Eur. J. Lipid Sci. Technol., 2011, 113, 616.

31 K. Dietliker, R. Hüsler, J.-L. Birbaum, S. Ilg, S. Villeneuve, K. Studer, T. Jung, J. Benkhoff, H. Kura, A. Matsumoto and H. Oka, Prog. Org. Coat., 2007, 58, 146.

32 J. Regensburger, T. Maisch, A. Knak, A. Gollmer, A. Felgenträger, K. Lehner and W. Bäumler, Phys. Chem. Chem. Phys., 2013, 15, 17672.

33 J. Regensburger, A. Knak, T. Maisch, L. Landthaler and W. Bäumler, Exp. Dermatol., 2011, 21, 135.

34 G. Kaur, P. Johnston and K. Saito, Polym. Chem., 2014, 5, 2171.

35 R. Wang and T. P. Schuman, eXPRESS Polym. Lett., 2013, 7, 272.

36 S. W. Benson, Chem. Educ. Eur., 1965, 42, 502. 